\title{
Management of Dental Esthetics in a Child with Hypodontia Using a Heat Vacuum-pressed Transparent Thermoplastic Polypropylene Template
}

\author{
S Nagarajan MP Sockalingam ${ }^{1}$, Ahmad SI Zakaria², Alida Mahyuddin ${ }^{3}$, Elavarasi Kuppusamy ${ }^{4}$
}

\begin{abstract}
Aim and objective: This case aimed to describe the use of a heat vacuum-pressed transparent thermoplastic polypropylene template to reconstruct dental esthetics in a growing child with hypodontia.

Background: Dental esthetics plays an integral part in the general well-being of children. The absence of a few teeth during the growing phases of life, especially during the teenage period, can interfere with the children's quality of life. Congenital absence of teeth is common in our society and often leads to dental problems such as spacing between teeth, malocclusion, and dental esthetics.

Case description: This case addressed the dental esthetics concern of a 12-year-old girl with hypodontia of her bilateral maxillary permanent lateral incisors using a vacuum-pressed thermoplastic polypropylene template with composite resin. The template helped to recontour the existing teeth morphology and enabled space closure between teeth.

Conclusion: The use of the thermoplastic polypropylene template method is less invasive, inexpensive, produced excellent dental esthetics, and suitable for growing children.

Clinical significance: This article highlighted the use of a heat vacuum-pressed transparent thermoplastic polypropylene template that may provide a viable solution to address the dental esthetics of multiple teeth in growing children. Using the template allowed quick recontouring of multiple teeth with composite resin during a single visit. The template also enabled quick polishing of the restorations because the good adaptation of the material to teeth means less trimming of excessive composite resin needed.

Keywords: Crown form, Dental esthetics, Dental anomalies, Hypodontia, Psychological, Template, Well-being.

World Journal of Dentistry (2021): 10.5005/jp-journals-10015-1843
\end{abstract}

\section{INTRODUCTION}

Dental esthetics influence the psychological and social well-being of children. 'Deficiency in dental esthetics affects children's social interactions negatively. ${ }^{2}$ Children as young as 6 years old are aware and able to differentiate between what they perceive as good sets of teeth. ${ }^{3}$ Self-image is important for children because they can look and feel good about themselves. Parental concerns play an important role in the dental esthetics of children. ${ }^{4}$ Parents want their children to look desirable in the eyes of others. Poor dental esthetics, especially noticeable dental differences and dental appearance were associated with the worst oral health-related quality of life in children. ${ }^{5}$

Several causes contribute toward poor dental esthetics of anterior teeth in children, namely size and shape of teeth, the color of teeth, black triangles, tooth position and spacing, malformed teeth, congenitally missing lateral incisors, and impacted canines. ${ }^{6}$ Many treatment modalities are available to address dental esthetic issues in children. These treatment options encompass both the non-invasive and invasive treatment procedures such as bleaching, microabrasion, direct and indirect composite restorations, direct and indirect composite veneers, porcelain veneers, orthodontics, porcelain crowns, and implants. ${ }^{7}$ Selection of an appropriate procedure to fix the dental esthetic issues depends on the clinical assessment of the tooth and dentition, expected desired outcome of the patient and parents, patient's age, and affordability of the treatment.

\begin{abstract}
${ }^{1-4}$ Department of Family Oral Health, Faculty of Dentistry, National University of Malaysia (UKM), Jalan Raja Muda Abdul Aziz, Kuala Lumpur, Malaysia

Corresponding Author: S Nagarajan MP Sockalingam, Department of Family Oral Health, Faculty of Dentistry, National University of Malaysia (UKM), Jalan Raja Muda Abdul Aziz, Kuala Lumpur, Malaysia, Phone: +60166606493, e-mail: drnaga67@gmail.com

How to cite this article: Sockalingam SNMP, Zakaria ASI, Mahyuddin A, et al. Management of Dental Esthetics in a Child with Hypodontia Using a Heat Vacuum-pressed Transparent Thermoplastic Polypropylene Template. World J Dent 2021;12(4):345-349.

Source of support: Nil

Conflict of interest: None
\end{abstract}

In the modern world, dental esthetics have become an integral part of general esthetics. Patients spend millions of dollars annually on dental esthetics in particular to boost their confidence and selfesteem. However, not all individuals have access to state-of-the-art technology in dentistry. However, there are methods and resources available to make esthetic dentistry available to those who could not afford expensive treatment.

This case report describes the management of esthetic concerns of a teenage girl from a lower-income family who has hypodontia using a cost-effective thermoformed vacuum-pressed transparent polypropylene crown-form shell for teeth shape modifications with dental composites. 


\section{Case Description}

\section{Clinical Presentation}

A healthy 12-year-old girl accompanied by her mother presented at the pediatric clinic with a concern about spacing between teeth. The girl was uncomfortable with the appearance of her teeth, and she has been ridiculed by some of her schoolmates. Before this visit, she had an appointment with an orthodontist who suggested fixed orthodontic treatment to close the spacing. However, the mother was not ready neither financially nor willing to commit to long-term treatment for her child. They wanted a reasonably quick and affordable treatment.

The girl's medical history did not reveal any significant findings. She was healthy with no known drug or food allergies. Her vaccinations were up to date. She weighed $35 \mathrm{~kg}$ and her height was $140 \mathrm{~cm}$. Extraoral examination showed she has a class I facial profile with a symmetrical face and competent lips. Her mouth opening was $39 \mathrm{~mm}$ and there was no deviation of the mandible on opening. She has an average smile with $75 \%$ exposure of the upper incisors.

Intraorally, she was in her permanent dentition with retained left maxillary primary canine (tooth 63 ) and congenitally missing right and left maxillary lateral incisors (tooth 12 and tooth 22). The maxillary permanent central incisors appear square (Fig. 1). Familial history of congenitally missing teeth was noted on the maternal side. She has good oral hygiene and healthy gingivae with no active caries. Tooth 63 was firm with a sound crown structure. The previous dental panoramic tomography (DPT) taken 15 months earlier at a dentist showed congenitally missing tooth 12 and tooth 22, and tooth 63 with good root length, bone height, and no periapical pathology (Fig. 2).

An occlusal assessment revealed molars in class I relationship on both sides, overjet of $2 \mathrm{~mm}$ and overbite of $75 \%$. Collectively $9.5 \mathrm{~mm}$ of space was available in the upper labial segment, with $5 \mathrm{~mm}$ on the right side and $4.5 \mathrm{~mm}$ on the left. The lower midline shifted $1.5 \mathrm{~mm}$ to the left.

Based on the assessment, treatment options were discussed with the patient and her mother. Both opted for the composite resin crown build-up treatment option. The overall treatment aimed to restore dental esthetics and enhance the self-esteem of the patient.

The entire procedure was carried out by a pediatric dentist. Alginate (Algeniux ${ }^{\circledR}$ Major) impressions of the maxillary and mandibular dentition were taken for study casts. Diagnostic set-up of the teeth was made on the stone cast with some composite material in the dental laboratory to facilitate communication with the patient and mother, and to assess the expected outcome of the shapes of the teeth extraorally (Fig. 3).

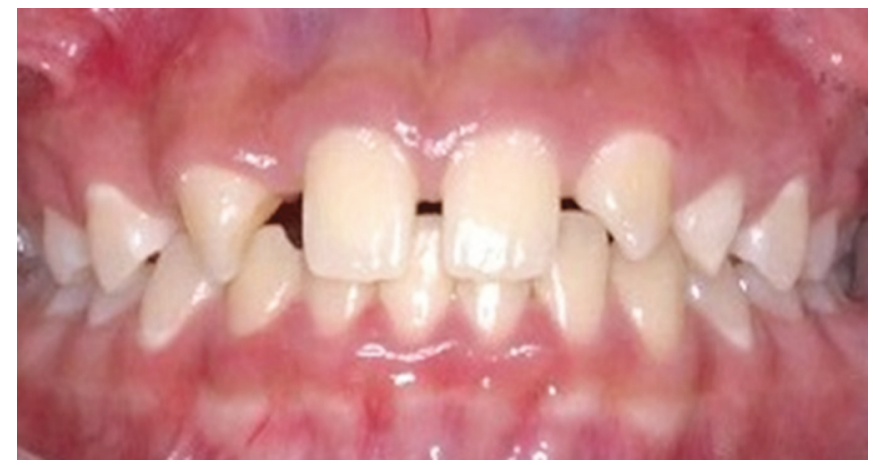

Fig. 1: Preoperative view of the dentition before treatment
After the acceptance of the expected final shape of the teeth, the stone cast model was used to construct a transparent plastic crown-form shell. A vacuum thermoforming machine (Essix ${ }^{\circledR}$ Machine, Dentsply Sirona) was used to construct a plastic crownform shell. The machine was preheated for 3 minutes until the heating element turns orange in color. A $1 \mathrm{~mm}$ thickness transparent thermoplastic polypropylene (Essix ACE ${ }^{\circledR}$ Plastic, Dentsply Sirona) sheet was placed into the frame of the machine and the stone cast model on the base plate. The preheated element was swung over the frame that held the thermoplastic sheet. As the plastic sheet sagged under the heat, the vacuum was turned on and the soft thermoplastic was lowered quickly over the stone cast model. The vacuum was kept continuously on until the plastic cooled. The vacuum-pressed thermoplastic sheet was removed from the model and trimmed using a pair of scissors (Essix ${ }^{\circledR}$ Mayo scissors, Dentsply Sirona) to fit the gingival contour (Fig. 4).

Both the permanent canines were build-up first using the plastic crown-form shell (Fig. 5). Both tooth 11 and tooth 21 were isolated with Teflon tape to prevent any adhesion of the resin. The tooth surfaces of the teeth that need composite build-up were roughened with a fine-grit diamond bur on the labial and the proximal sides. The prepared surfaces were etched with $37 \%$ phosphoric acid (N-Etch Jumbo ${ }^{\circledast}$ Ivoclar, Vivadent AG) for 20 seconds and later washed with a copious amount of water and blow-dried. A bonding agent (Adper ${ }^{\circledast}$ Single Bond, 3M ESPE) was applied to the etched dried surfaces and light-cured for 20 seconds. Two tiny holes were made using a small round bur on the palatal aspect of the crownform shell to act as air vents. Composite resin (Filtek Z350XT ${ }^{\mathrm{TM}}, 3 \mathrm{M}$ ESPE) was placed into the crown-form shell that corresponds to the teeth that needed crown build-up. The composite resin-filled crown-form shell was gently placed over teeth for adaptation of the composite resin. Light cure was shone through the transparent crown-form shell to harden the composite. After curing, the crownform shell was and the excess composite on the build-up teeth was trimmed and the teeth polished. The occlusion checked for lateral excursions and forward protrusion to eliminate and heavy contacts. The patient was happy with the final appearance of the modified shape of the teeth (Fig. 6). The restorations were intact and the dental esthetics were unaffected at 1-year follow-up.

\section{Discussion}

Hypodontia is defined as the congenital absence of six or fewer teeth primary or permanent teeth in the dentition, excluding the third

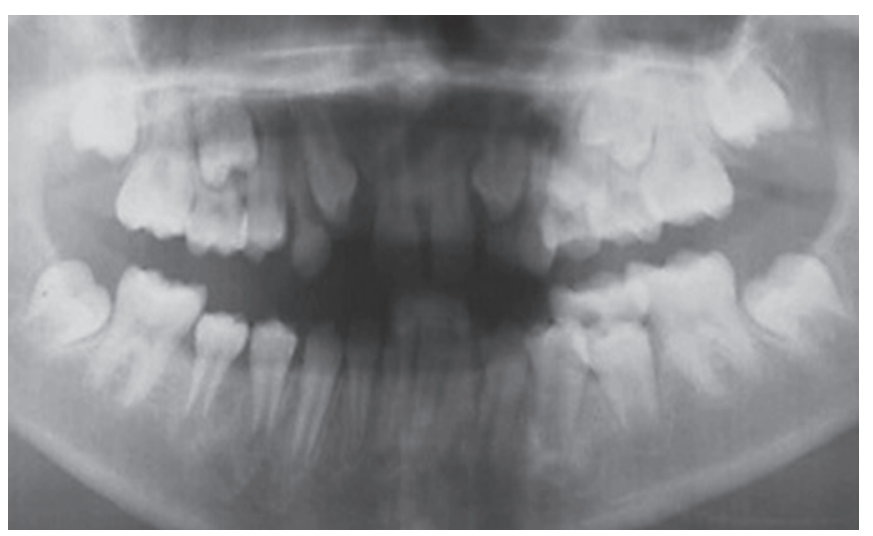

Fig. 2: Dental panoramic tomography (DPT) taken 15 months before treatment showing missing permanent maxillary lateral incisors 


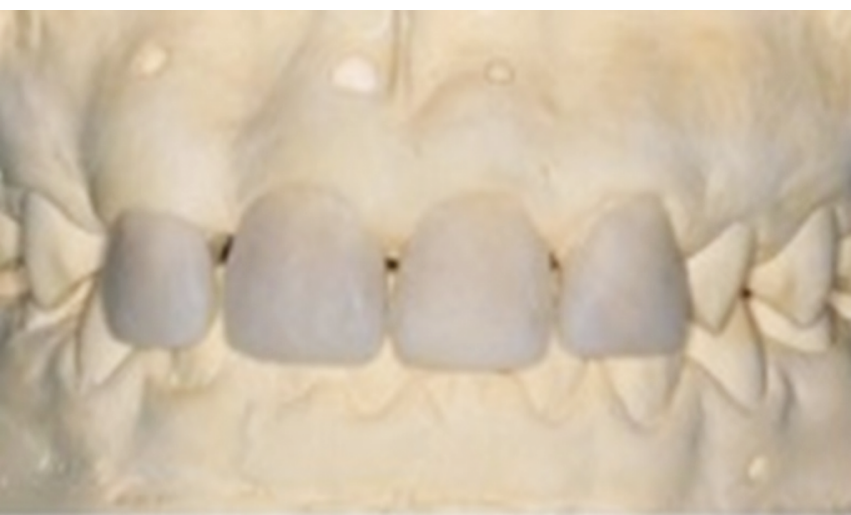

Fig. 3: Diagnostic set-up of the teeth using composite on a stone cast

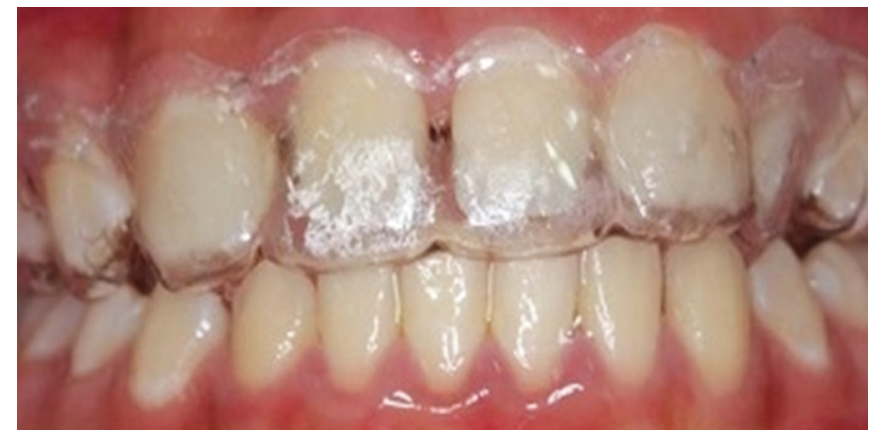

Fig. 5: Trimmed transparent thermoplastic polypropylene template placed over the teeth to be reshaped

molars. ${ }^{8}$ Its prevalence varies between $1.6 \%$ and $6.9 \%$ depending on the studies. ${ }^{9}$ Most people have one or two congenitally missing teeth, and maxillary lateral incisors are one of the commonly affected teeth besides permanent second premolars. ${ }^{10}$ Severe hypodontia is often associated with some underlying systemic conditions such as ectodermal dysplasia, Down syndrome, incontinentia pigmenti, Witkop syndrome, and Rieger syndrome. ${ }^{11}$ In the maxillary anterior region, agenesis of permanent lateral incisors is greater compare to other anterior teeth with some cases showing bilateral agenesis and higher female preponderance. ${ }^{12,13}$ Although the etiology of hypodontia is multifactorial, a familial trend with genetic inheritance may contribute. ${ }^{10,14}$ In the present case, both the patient's mother and one of her siblings also had congenitally missing maxillary permanent lateral incisors bilaterally.

Malalignment or palatal impaction of the erupting permanent canine, retained predecessor's tooth, spacing in the labial segment, median diastema, and microdontic or peg-shaped contralateral permanent lateral incisor are some clinical issues seen in individuals missing permanent maxillary lateral incisors. ${ }^{15,16}$ In the present case, spacings between teeth with median diastema, retained primary canine (tooth 63 ) and square-shaped permanent incisors were noted. The spacing between teeth occurred because of the discrepancy between the number of teeth and the amount of space available. The space available in the labial segment was greater than the sum of the mesiodistal width of both the maxillary permanent incisors and canines. Both tooth 13 and tooth 23 have erupted into the spaces of lateral incisors with failure of physiological root resorption of tooth 63 on the left side because of the altered eruption path of tooth 23 .

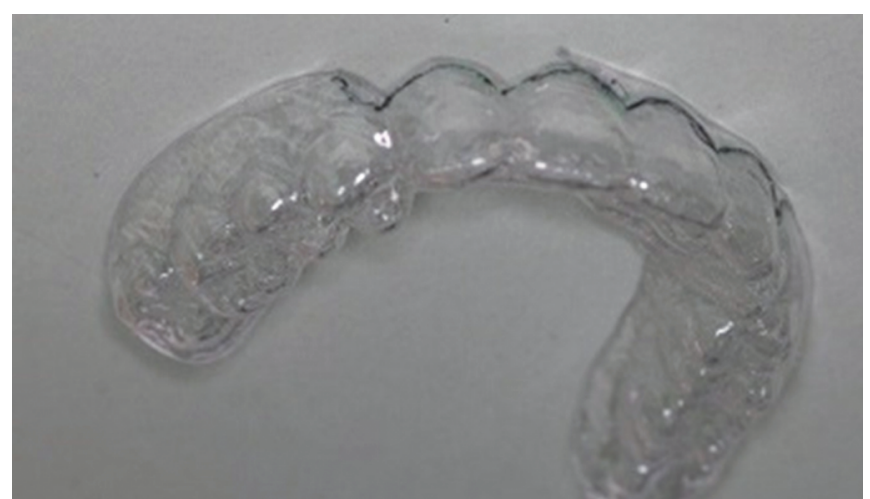

Fig. 4: Vacuum-pressed transparent thermoplastic polypropylene template trimmed to fit the gingival contour

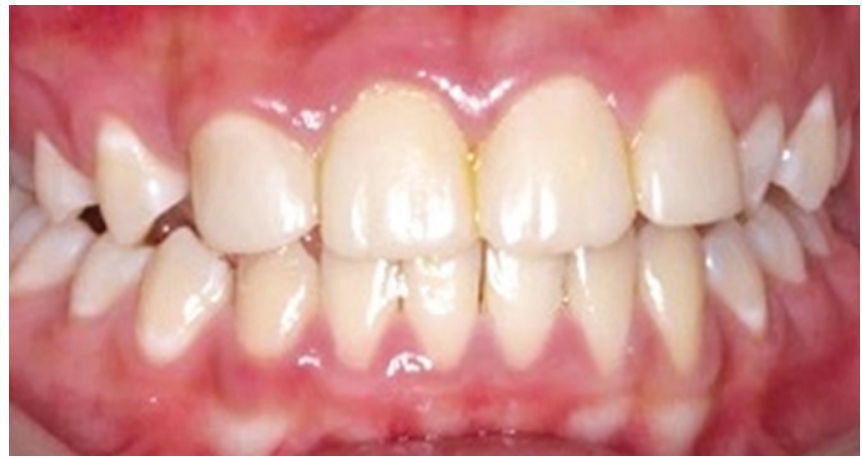

Fig. 6: Postoperative outcome of the modifications of the teeth shape and size using composite place into the transparent thermoplastic polypropylene template

Treatment modalities to address hypodontia cases vary from simple to complex procedures. The treatment ranges from acceptance of retained teeth and spaces to space redistribution either by opening the spaces available or closing them. Space closure can be either achieved by moving the canine tooth into the lateral incisor space orthodontically or tooth shape modifications using adhesive composites or ceramic materials. ${ }^{17,18}$ Space opening allows redistribution of spaces to accommodate prostheses such denture, adhesive bridge, implant, or the created space can be used for tooth transplantation. ${ }^{17-19}$ Whatever the treatment option, several factors such as patient's concern and parental expectations, patient's age, teeth exposure during a smile, the severity of the hypodontia, teeth-space availability in the arch, occlusion, oral hygiene, and affordability of the treatment need to be taken into consideration. Besides, in growing patients, issues such as short clinical crown height, large pulp chambers, immature gum height, a transition of dentition, and jaw growth may favor a more conservative or minimally invasive treatment so that the longterm integrity of the tooth can be preserved for more esthetic and invasive treatment in adulthood.

In the current case, the patient and her mother opted for modification of teeth with adhesive composite to reshape the maxillary permanent central incisors and canines and to retain the primary canine (tooth 63). This treatment option has several limitations such as wearing and discoloration of composite over time and altering gum esthetic in growing patients. Also, it is not possible to close $100 \%$ of the spaces available through teeth 
modifications because this may cause an unesthetic appearance of the teeth due to disharmony in the mesiodistal width and incisalgingival height ratio. This treatment option is minimally invasive, preserves tooth structure, affordable, and most viable in growing patients.

A diagnostic cast setup of the teeth that need modification using composites was helpful to provide the clinician with a three-dimensional assessment of spaces available and occlusion, to forecast the expected outcomes of the treatment, and to facilitate communication with the patient and her mother. The final diagnostic cast was used to prepare the vacuum-pressed transparent thermoplastic polypropylene sheet crown form. The material is thin but firm upon cooling, easy to trim with scissors to fit the gum margin, and custom-made to fit the desired crown size.

Advancements in composite technology such as a variety of tooth shades, predictable tooth-color stability, minimal material shrinkage, high compressive, and flexural strength coupled with high polishability have enabled us to use this material widely in dentistry. Adhesive dentistry has facilitated clinicians to provide quality dentistry at an affordable cost to patients, especially to those from the middle- and lower-income groups. Although the finesse in the details may lack, the patients' satisfaction is guaranteed. In the current case, the patient is from a lower-income family and she cannot afford expensive dental treatment. The outcome of the treatment has given the patient pleasing dental esthetics and boosted her self-confidence.

Adhesive composites can modify crown shapes either as a direct or an indirect restoration. Direct composite restoration can be made using a freehand technique, cellulose acetate crown-form, preformed crown template facing, or placement of the commercially available pre-fabricated composite veneer. ${ }^{20-22}$ Indirect composite restoration such as custom-made composite veneer can be fabricated in the laboratory to fit the patients' needs. ${ }^{23}$ In the current case, we used thermoformed vacuum-pressed transparent polypropylene crown-form shell to modify the dimension of the teeth and correct the spaces between the teeth. ${ }^{24}$ This method is useful when dealing with children who need multiple teeth modifications and fewer visits were necessary for the completion of treatment. The prepared crown form can be reused during the subsequent visits. The strength and flexibility of the constructed crown form allow easy placement and removal from the tooth with little distortion and tearing.

\section{ConcLusion}

The thermoformed vacuum-pressed transparent polypropylene crown-form shell is a suitable tool to use in growing children who need multiple teeth modifications and space closures. The crown form is simple to construct, cost-saving, size, and shape of teeth easy to reproduce and it has dimensional stability to allow reusability.

\section{Clinical Significance}

One of the challenges faced by dental clinicians is providing a definitive solution for dental esthetics concerns in growing children, especially young adolescents. Often jaw growth, changing occlusion, immature gum heights, and treatment compliance may dictate the treatment planning. The use of a heat vacuum-pressed transparent thermoplastic polypropylene template provides a viable solution to address the dental esthetics of multiple teeth in growing children. The template allows quick recontouring of multiple teeth with composite resin during a single visit. The template also enables quick polishing of the restorations because the good adaptation of the composite resin material to teeth means less trimming of the excessive material needed.

\section{References}

1. Gupta T, Sadana G, Rai HK. Effect of esthetic defects in anterior teeth on the emotional and social well-being of children: a survey. Int J Clin Pediatr Dent 2019;12(4):229-232. DOI: 10.5005/ jp-journals-10005-1628.

2. Rossini G, Parrini S, Castroflorio T, et al. Children's perceptions of smile esthetics and their influence on social judgment. Angle Orthod 2016;86(6):1050-1055. DOI: 10.2319/102715-722.

3. Pani SC, Saffan AA, AlHobail S, et al. Esthetic concerns and acceptability of treatment modalities in primary teeth: A comparison between children and their parents. Int J Dent 2016. DOI: 10.1155/2016/ 3163904.

4. Dave BH, Shah VU, Bargale S, et al. Evaluation of parental perception regarding smile of children according to visual analog scale. J Integr Health Sci 2018;6(2):41-44. DOI: 10.4103/JIHS.JIHS_16_18.

5. Rodd HD, Marshman Z, Porritt J, et al. Oral health-related quality of life of children in relation to dental appearance and educational transition. Br Dent J 2011;211(2):E4. DOI: 10.1038/sj.bdj.2011.574.

6. Bartlett D. Aesthetic problems. Pocket dentistry, ch. 5 2020. pp. 55-62. Available from: https://pocketdentistry.com/5-aesthetic-problems/.

7. Bryan RA, Welbury RR. Treatment of aesthetic problems in paediatric dentistry. Dent Update 2003;30(6):307-313. DOI: 10.12968/ denu.2003.30.6.307.

8. Rakhshan V.Congenitally missing teeth (hypodontia): A review of the literature concerning the etiology, prevalence, risk factors, patterns and treatment. Dent Res J (Isfahan) 2012;12(1):1-13. DOI: 10.4103/17353327.150286.

9. Al-Ani AH, Antoun JS, Thomson WM, et al. Hypodontia: An update on its etiology, classification, and clinical management. Bio Med Res Int 2017;2017:9378325. DOI: 10.1155/2017/9378325.

10. Arte S, Hypodontia PS, Orphanet 2004. [cited 28 December 2020]. Available from: http://www.orpha.net/data/patho/GB/ uk-hypodontaia.pdf.

11. Shimizu T, Maeda T. Prevalence and genetic basis of tooth agenesis. Jpn Dent Sci Rev 2009;45(1):52-58. DOI: 10.1016/j.jdsr.2008.12.001.

12. Fujita Y, Hidaka A, Nishida I, et al. Developmental anomalies of permanent lateral incisors in young patients. J Clin Pediatr Dent 2009;33(3):211-215. DOI: 10.17796/jcpd.33.3.c74j49r738676671.

13. Arandi NZ, Mustafa S. Maxillary lateral incisor agenesis; a retrospective cross-sectional study. Saudi Dent J 2018;30(2):155-160. DOI: 10.1016/j. sdentj.2017.12.006.

14. Cobourne MT. Familial human hypodontia. Br Dent J 2007;203(4):203208. DOI: 10.1038/bdj.2007.732.

15. PinhoT, Maciel P, Pollmann C. Developmental disturbances associated with agenesis of the permanent maxillary incisors. Br Dent J 2013;207(12):E25. DOI: 10.1038/sj.bdj.2009.961.

16. Yadav S, Upadhyay M, Uribe F, et al. Palatally impacted maxillary canine with congenitally missing lataeral incisors and midline diastema. Am J Orthod Dentofac Orthop 2013;144(1):141-146. DOI: 10.1016/j.ajodo.2012.05.023.

17. Paduano S, Cioffi I, Rongo R, et al. Orthodontic management of congenitally missing lateral incisors: A case report. Case Rep Dent 2014. DOI: 10.1155/2014/731074.

18. Abdulgani A, Watted N, Abu-Hussein M. Congenitally missing lateral incisors, orthodontic, restorative and implant approaches. Int J Dent Oral Health 2016;2(3):24-33. DOI: 10.25141/2471-657X-20163.0071.

19. Hariri R, Alzoubi EEM. Autotransplantation in combination with orthodontic treatment. J Orthod Sci 2019;8(1):11. DOI: 10.4103/jos. JOS_62_18. 
20. Mackenzie L. Direct anterior composites: A practical guide. Dent Update 2013;40(4):297-317. DOI: 10.12968/denu.2013.40. 4.297.

21. Lowe RA, Creating beautiful restorations in the aesthetic zone. [cited on 30 December 2020]. Available from: https://www.dentistrytoday. com/articles/10060.

22. Lewis MW, Braxton AD, Wasson W, Prefabricated composite veneers: a conservative solution for aesthetic zone. [cited on 30
December 2020]. Available from: https://www.dentistrytoday.com/ restorative-134/10101.

23. Gargari M, Ceruso FM, Pujia A, et al. Restoration of anterior teeth using an indirect composite technique. Case report Oral Implantol (Rome 2013;6(4):99-102.

24. Sockalingam SNMP. Dental rehabilitation of amelogenesis imperfecta using thermoformed templates. J Indian Soc Pedod Prev Dent 2011;29(1):53-56. DOI: 10.4103/0970-4388.79938. 